SPIE Paper No. 4448-16

\title{
Projection Moiré Interferometry Measurements of Micro Air Vehicle Wings
}

Gary A. Fleming, Scott M. Bartram, Martin R. Waszak, and Luther N. Jenkins

National Aeronautics and Space Administration

Langley Research Center

Hampton, Virginia, 23681-2199, USA

Presented at the SPIE conference on Optical Diagnostics for Fluids, Solids, and Combustion, part of the SPIE International Symposium on Optical Science and Technology,

San Diego, CA, July 29 - August 3, 2001. 


\title{
Projection Moiré Interferometry Measurements of Micro Air Vehicle Wings
}

\author{
Gary A. Fleming, Scott M. Bartram, Martin R. Waszak, and Luther N. Jenkins \\ National Aeronautics and Space Administration \\ Langley Research Center \\ Hampton, Virginia, 23681-2199 USA
}

\begin{abstract}
Projection Moiré Interferometry (PMI) has been used to measure the structural deformation of micro air vehicle (MAV) wings during a series of wind tunnel tests. The MAV wings had a highly flexible wing structure, generically reminiscent of a bat's wing, which resulted in significant changes in wing shape as a function of MAV angle-of-attack and simulated flight speed. This flow-adaptable wing deformation is thought to provide enhanced vehicle stability and wind gust alleviation compared to rigid wing designs. Investigation of the potential aerodynamic benefits of a flexible MAV wing required measurement of the wing shape under aerodynamic loads. PMI was used to quantify the aerodynamically induced changes in wing shape for three MAV wings having different structural designs and stiffness characteristics. This paper describes the PMI technique, its application to MAV testing, and presents a portion of the PMI data acquired for the three different MAV wings tested.
\end{abstract}

Keywords: Projection Moiré Interferometry, PMI, micro air vehicle, MAV, aeroelastic, wing deformation

\section{INTRODUCTION}

A number of research organizations have been investigating biologically inspired flight systems (BIFS) and micro air vehicles (MAV) since the mid-1990s. The interest in these aircraft, which are nominally $150 \mathrm{~mm}$ in their largest dimension and fly at or below speeds of $\sim 40 \mathrm{~km} / \mathrm{h}^{1}$, has been primarily driven by the Defense Advanced Research Projects Agency (DARPA) who is pursuing MAVs for use in military missions such as reconnaissance and collaborative munitions deployment. Conceptually, these small, expendable aircraft could be launched by ground troops and be either remotely or autonomously piloted to conduct surveillance in areas of extreme hazard, such as battlefield regions contaminated with biological / chemical warfare agents. MAVs could also be used in an urban warfare environment to conduct reconnaissance in areas inaccessible to larger aircraft, such as areas within buildings, to identify and segregate military targets versus areas having high civilian concentrations. Many civilian and commercial applications also exist for MAVs, including traffic monitoring, accident assessment, and wildlife / land management.

Several universities and commercial companies ${ }^{2}$ are pursuing independent MAV designs that encompass many of the characteristics necessary to satisfy the requirements for both military and civilian applications. At least two universities, Arizona State University and the University of Florida, host MAV design and flight competitions. The goals of the competitions are to conduct target identification and aerial surveillance on a target positioned on the ground 600 meters from the launch site, and to fly a 57 gram payload for a duration of 2 minutes $^{3}$. Although these competition goals may seem simplistic, they are representative of the missions that may be encountered in future military and civilian applications and prove to be a challenge for vehicles within the MAV class.

In 2000, NASA Langley Research Center began a research effort to investigate the aerodynamics of BIFS and MAVs for the purposes of understanding the biological flight mechanisms that could be exploited for other types of aircraft. Rather than design and fabricate an original MAV from scratch, existing MAVs were considered for detailed aerodynamic evaluation and modeling. One configuration chosen for study was based on a vehicle originally developed by a team of 
researchers at the University of Florida (UF), whose MAVs have won the International Society of Structural and Multidisciplinary Optimization (ISSMO) Micro Aerial Vehicle Competition for the past three years ${ }^{3,4}$. A vehicle based on the UF design, but adapted for aerodynamic testing, is shown in figure 1. The aircraft is unique in that it has a highly flexible wing structure generically reminiscent of a bat's wing. The wing skeletal members, consisting of a leading edge spar and chordwise battens, are fabricated from lightweight carbon fiber laminate. The wing skin is a flexible, thin membrane, typically $0.1 \mathrm{~mm}$ thick latex. At this thickness, the latex membrane is 4 to 5 times thinner than a standard surgical glove. The wing mounted to the MAV shown in figure 1 is a $152 \mathrm{~mm}$ full span "single batten" wing, as it has one batten per semispan wing section. Numerous other wing constructions have been evaluated during the course of UF and NASA testing, ranging from 1 to 6 battens per semispan wing section with different skin materials having

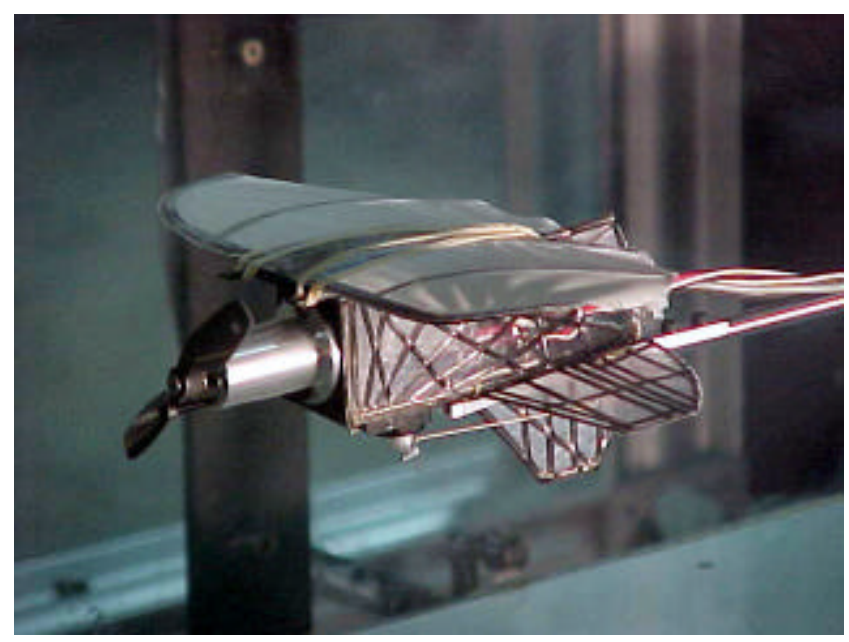

Figure 1: University of Florida MAV design adapted for aerodynamic testing varying stiffness properties. The MAV fuselage is designed to be rigid and fabricated from a graphite-epoxy material covered with a thin monofilm membrane. The MAV is powered using a small gas engine during actual flights, but a DC electric motor was implemented for wind tunnel testing. Further details of the vehicle construction can be found in reference 4 .

The primary benefit of the aeroelastic wing is that its flexibility allows it to deform locally to react to wind gusts or other spontaneous changes in flight condition, leading to a more stable, controllable flight. The aeroelastic wing has also been shown to delay stall at high angles-of-attack (AoA) compared to rigid wings ${ }^{5}$, thus increasing the vehicle flight envelope. One unfortunate consequence of the aeroelastic wing is, however, that the non-constant wing geometry makes the vehicle flight dynamics difficult to understand and model. Therefore, a series of wind tunnel tests were conducted at the NASA Langley Basic Aerodynamics Research Tunnel (BART) in September, 2000 and March, 2001. The purpose of these tests was to collect aerodynamic stability and control data for UF-designed MAVs, and to investigate how these data were related to different aeroelastic wing constructions. Achieving these objectives required strain gauge balance measurements for determination of the vehicle aerodynamic forces and moments, and the use of Projection Moiré Interferometry (PMI) for quantifying the aeroelastic wing deformation. Further details of the aerodynamic data and analysis can be found in reference 5, and will not be addressed here. The focus of this paper is to describe the PMI technique, its application to MAV testing, and to present sample results obtained throughout the course of wind tunnel testing at NASA Langley.

\section{PROJECTION MOIRÉ INTERFEROMETRY (PMI)}

PMI is a video-based, non-contacting, non-intrusive measurement technique capable of obtaining spatially continuous measurements of out-of-plane structural deformations. The PMI technique has been under development at NASA Langley since 1996 for acquiring deformation measurements of wind tunnel models under aerodynamic loads. During this course of development, PMI has been used in several large scale wind tunnel facilities to measure deformations of spinning rotorcraft blades ${ }^{6-7}$, fixed wing, and semispan ${ }^{8}$ aircraft models. Further details of the development program can be found in reference 9 .

PMI as applied to measuring wind tunnel model deformation is shown schematically in figure 2. A projection system is used to project a grid of equispaced, parallel lines onto the wind tunnel model surface. Any incoherent light source providing adequate illumination levels can be used for grid line projection, but pulsed laser diodes emitting in the near infrared (IR) are often used for large scale wind tunnel tests. Laser diodes provide the high light intensity levels required for measuring deformations over large fields-of-view, and prevent the projected grid line contrast from being washed out 


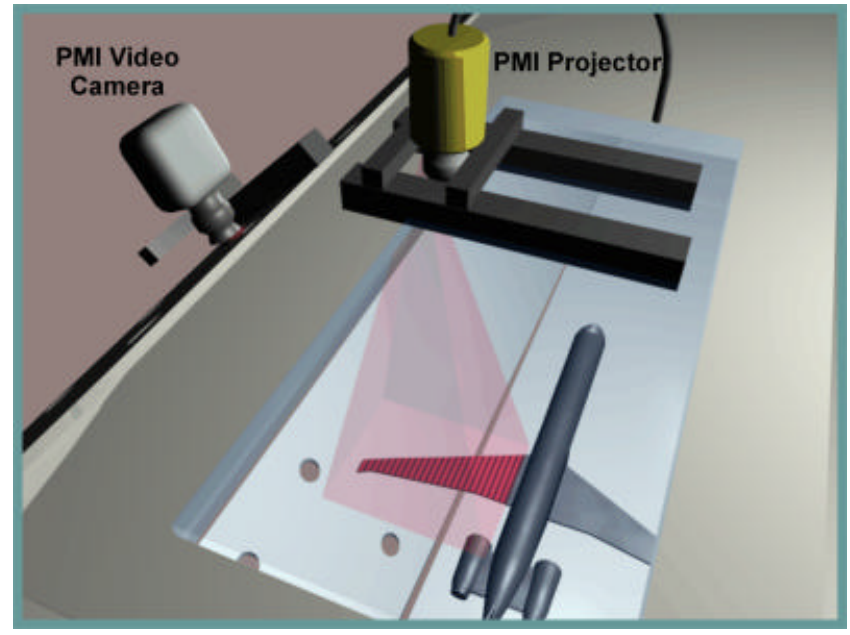

Figure 2: Conceptual use of PMI for wind tunnel model deformation measurements

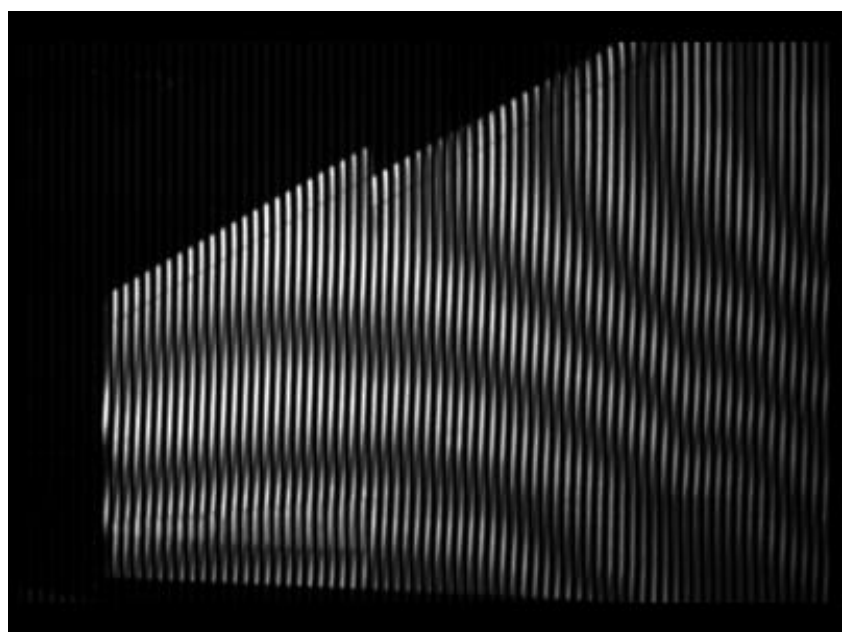

Figure 4: Moiré fringes generated by interfering raw PMI data, figure 3 , with a computationally generated reference grid

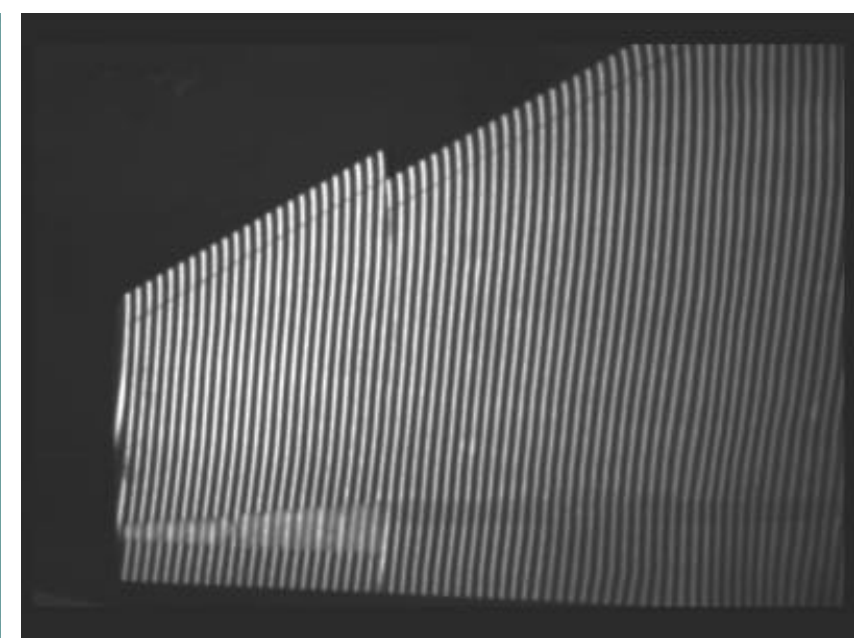

Figure 3: Raw PMI data image showing projected grid lines

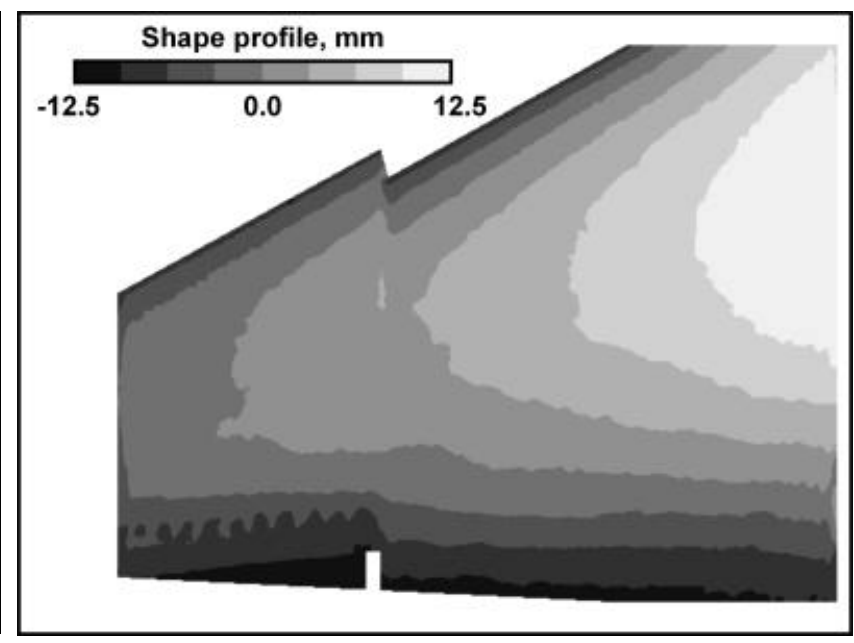

Figure 5. PMI-measured airfoil shape data obtained by processing moiré fringe images as in figure 4

by ambient test section lighting if an appropriate narrow band pass filter is used on the PMI system video camera. This allows for lights-on tunnel operation, alleviating many safety concerns. The projector system is typically aligned such that its optical axis is perpendicular to the surface being measured. A Ronchi ruling (a transmissive grating with opaque parallel lines etched at equal spacing and thickness) installed in the projector is the physical element generating the projected grid lines. A CCD camera with a narrow band pass filter matched to the projector illumination wavelength is positioned to view the model at a $30-45^{\circ}$ angle inclined from the projector optical axis. The projector and camera must lie within a plane perpendicular to the projected grid lines. Images of the grid lines projected onto the model are acquired in baseline (wind-off) and loaded (wind-on) conditions using a frame grabber installed in a PC-compatible computer. An example PMI raw data image with an approximate 1.2-x 1.2-meter field-of-view is shown in figure 3. Image processing routines are then used to remove camera perspective distortion and interfere the acquired images with a computationally generated reference grid, resulting in interferograms containing moiré fringes, figure 4 . These fringe patterns are further processed offline to obtain a quantitative, spatially continuous representation of the model surface shape or deformation, as shown in figure 5. The PMI system measurement accuracy and resolution are primarily dependent upon the projected grid pitch, video camera field-of-view, optical modulation transfer function, and illumination / observation angles ${ }^{10}$. The deformation measurement accuracy and resolution of the PMI system used to 
acquire the data shown in figures 3-5 was $0.75-\mathrm{mm}$ with $0.5-\mathrm{mm}$ resolution ${ }^{8}$, typical of PMI systems used in other large wind tunnel applications.

\section{PMI APPLIED TO MAV TESTING}

The lack of rigidity of the MAV wing, particularly the wing skin, presented several measurement requirements that needed satisfied to obtain accurate aero-loaded wing deformation measurements. Non-uniform, asymmetric wing deformations were expected, rendering the need for global, spatially continuous measurements. The wing skin was also expected to exhibit a significant amount of unsteady deformation from mid-chord to the trailing edge occurring at frequencies of several hundred hertz, and measurements of both the mean and RMS values of this unsteady deformation were of interest. Finally, any measurement method applied needed to be completely non-contacting as to not disturb the wing surface or alter its dynamics, and non-intrusive to preserve flow quality within the wind tunnel. The PMI technique satisfied all of these measurement requirements, and was therefore chosen as the primary method for acquisition of MAV wing deformation data throughout the course of wind tunnel testing.

\subsection{Experimental Configuration}

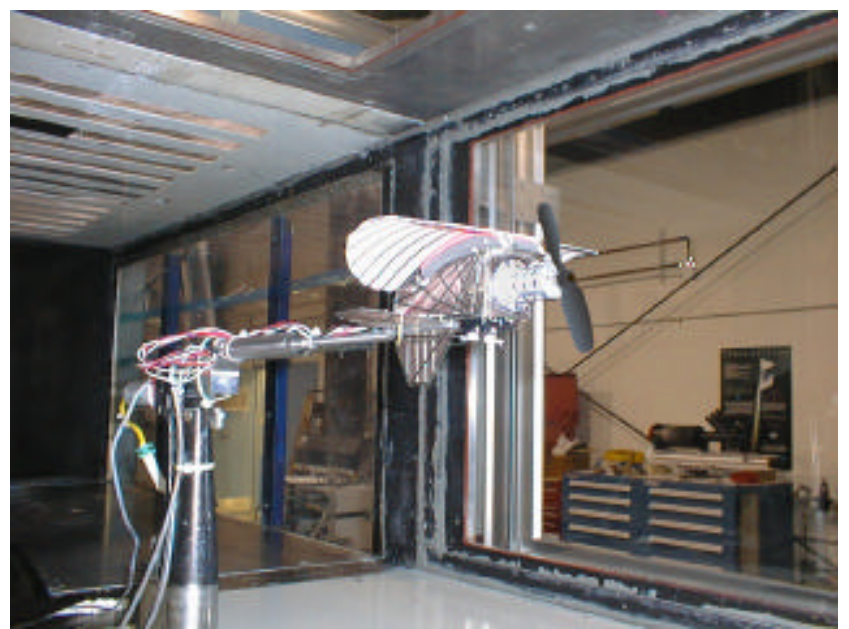

Figure 6: MAV installed in the NASA Langley BART

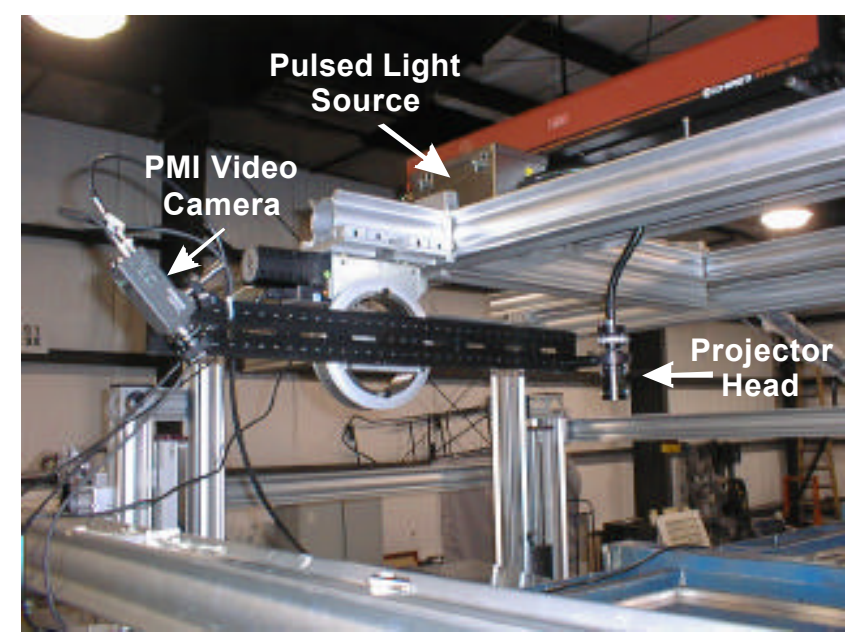

Figure 7: PMI system optical configuration

Figure 6 shows the MAV installed for aerodynamic testing in the NASA Langley BART facility. The BART is a flowdiagnostic facility that specializes in the acquisition of detailed data for the development and validation of CFD models and techniques. The BART is a subsonic, open-return wind tunnel with a closed test section 0.711 meters high, 1.016 meters wide and 3.048 meters long. The tunnel operates at atmospheric pressure up to a maximum free stream velocity of $56 \mathrm{~m} / \mathrm{s}$. Additional information about the BART can be found in references 11-13.

The PMI system optical configuration is shown in figure 7. A high intensity, fiber optically coupled pulsed light illuminator was used as the projector light source. The illuminator output 8-microsecond duration light pulses containing energy throughout the visible spectrum. The short pulse duration allowed the highly dynamic MAV wing motion to be effectively frozen during each light pulse, preventing blurring of the projected grid lines. The light was fiber optically coupled to a projector head containing a 15.75 line pairs $/ \mathrm{mm}$ Ronchi ruling. The resulting grid pattern was projected onto the MAV wing surface using a standard 8-48 $\mathrm{mm}$ zoom C-mount video lens set to an approximate $40 \mathrm{~mm}$ focal length. The projector was mounted above the test section ceiling, 1.0 meter above the MAV wing nominal horizontal axis. The projector head was aligned to project the grid pattern vertically downward onto the MAV wing top surface. The PMI system video camera was a 1300-x 1030 pixel progressive scan CCD camera with a standard $50 \mathrm{~mm}$ fixed focal length C-mount video lens. The camera was mounted upstream of the projector, angled at approximately $40^{\circ}$ 
downstream to view the top of the MAV wing. Video signals from the camera were transmitted via video fiber optic relays to a frame grabber board internal to a PC-compatible computer located in the tunnel control room. Use of the video fiber optic relays significantly reduced the noise observed on the video signal compared to transmission of the signal over standard analog video cable.

Timing and triggering signals were provided to the light source and camera to ensure a single light pulse occurred during the camera $100 \mu \mathrm{s}$ open shutter period. Multiple light pulses during the same exposure would produce images containing blurred grid lines, which cannot be quantitatively processed to determine wing shape. Additionally, the short exposure time minimized the presence of background light from ambient tunnel lighting in the PMI data images. Since a visible light source was being used as the projector illumination source, the projected light could not be isolated from the ambient lighting by optical filtering as would be the case if laser light was used for grid line projection.

The PMI system was calibrated by removing the MAV from the test section and inserting a flat aluminum honeycomb plate in a horizontal plane at the same elevation as the MAV wing leading edge. One side of the plate contained an array of black dots (approximately 30-x 30) on $6.35 \mathrm{~mm}$ centers painted on a white background. The side containing the dots was placed facing vertically upward to be viewed by the PMI system video camera. Images of the dot card illuminated by ambient lighting were digitized and processed to determine (a) the camera spatial resolution, and (b) image dewarping parameters necessary to computationally remove the video camera optical and perspective distortions. The camera spatial resolution was measured to be $0.22 \mathrm{~mm} /$ pixel. Following acquisition of the dot card images, the calibration plate was turned over so the opposing side, painted flat white, faced upward. Grid lines were projected on to the plate surface, and the plate was rotated about the horizontal axis perpendicular to the mean flow direction from $-10^{\circ}$ to $+10^{\circ}$ in $1^{\circ}$ increments. The plate rotation angles were measured to better than $0.01^{\circ}$ using a precision accelerometer. Images of the projected grid lines were captured at each incremental plate angle, and were later processed to determine the PMI system fringe sensitivity constant (FSC). Physically, if each moiré fringe in an interferogram (see figure 4) is thought of as a contour line of constant deformation, the FSC is the contour interval. The nominal FSC for the system used during these wind tunnel tests was $2.4 \mathrm{~mm} /$ fringe.

The MAV test matrix specified PMI and aerodynamic performance measurements to be acquired for the vehicle over a $-5^{\circ}$ to $+30^{\circ}$ AoA range. AoA changes in the BART resulted in vertical/streamwise motion of the model because the sting and model support mechanism present during the time of testing rotated around a pivot point that was not aligned with the model center of gravity. This is typically not a significant problem to PMI measurements for small AoA changes. However, the translation of the model over the prescribed AoA range would have caused the model to migrate out of the PMI system field-of-view if the PMI system were held stationary. Therefore a three dimensional automated
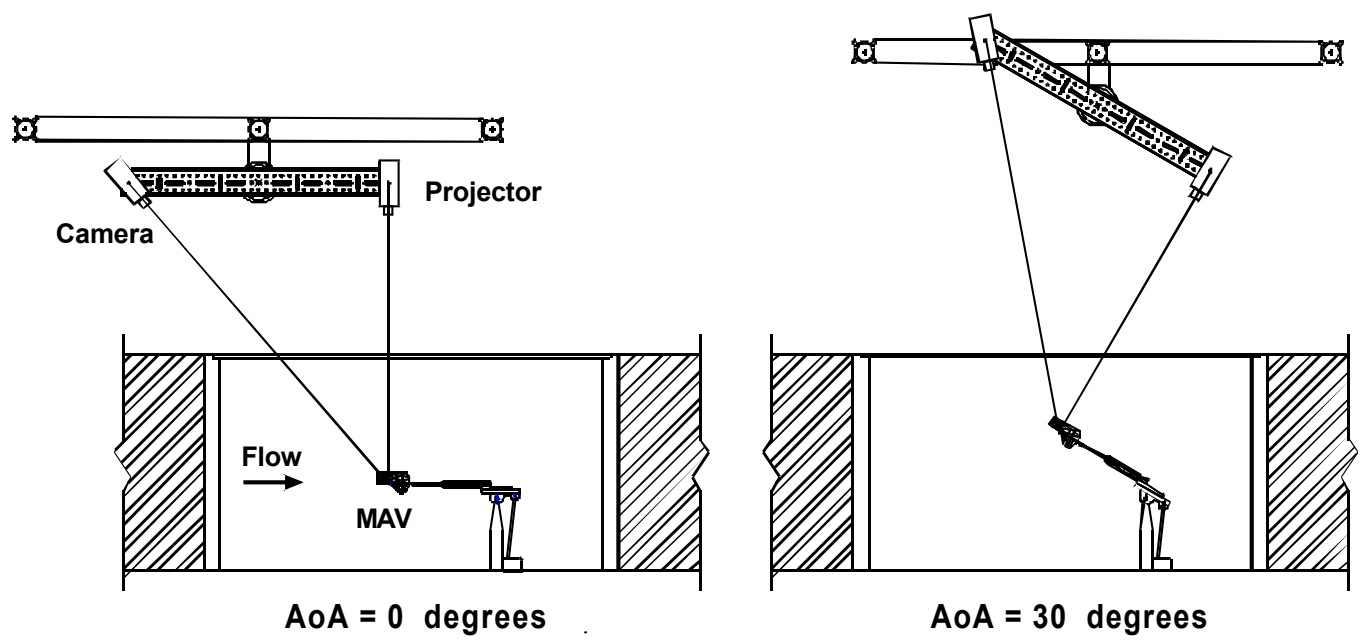

Figure 8: Schematic representation showing PMI system positioning as a function of MAV angle-of-attack 
positioning system resident at the BART was used to move the PMI system as a function of model AoA and compensate for the vertical/streamwise model motion. A schematic representation showing the PMI system positioning is shown in figure 8. A rotation stage was used in conjunction with the translation equipment to rotate the PMI system to keep the projector optical axis perpendicular to the wing surface. Repositioning the PMI equipment produced some minor inplane image shifting, approximately $7 \mathrm{~mm}$ over the full AoA range, caused by refractive effects of the $11 \mathrm{~mm}$ thick float glass tunnel ceiling window. This image shifting was removed by image pattern matching techniques applied during the PMI data processing. Laboratory experimentation and optical ray tracing performed prior to the start of wind tunnel testing showed the sensitivity of the PMI system remained constant while being positioned throughout the AoA range. This was also confirmed through analysis of the PMI data acquired during the tunnel tests.

\subsection{Data Acquisition and processing}

Data acquisition in the BART facility is a fully automated process, controlled by the BART data acquisition system (DAS). For example, an AoA sweep is accomplished by programming the DAS with the desired test angles, then commanding the DAS to begin the acquisition process. The DAS will automatically reposition the model to each specified angle, settle, acquire and store the relevant data, then continue to the next angle. The PMI data acquisition computer was networked with the BART DAS so that PMI data acquisition could also be performed automatically. This further ensured the PMI data were acquired during the same time period as the aerodynamic performance data. The BART DAS was also programmed to control the automated positioning system to reposition the PMI system as a function of the MAV AoA. Data were typically acquired by conducting AoA sweeps at a constant tunnel free stream velocity, in the following sequence: (a) move the MAV to the appropriate AoA, (b) reposition the PMI system, (c) acquire and store data, (d) continue to next angle.

PMI images of the MAV wing were captured at a resolution of 1300-x 1030 pixels (w-x h) at a data rate of $10 \mathrm{~Hz}$. The data rate was chosen arbitrarily, but was restricted to be less than the $12 \mathrm{~Hz}$ maximum frame rate of the camera. 100 PMI images were captured for each tunnel point, defined as a change in vehicle AoA, vehicle operating condition, or tunnel velocity. This size data set was chosen to achieve a statistically significant sampling and obtain sufficient averaging to determine accurate mean wing shapes in the presence of dynamic motion. PMI data acquisition began immediately after receiving a trigger signal from the BART DAS. The raw PMI image files were saved to disk, then further processed off-line to obtain wing deformation measurements. Each PMI data image within a 100-image set was processed independently. The 100 resulting deformation maps were subsequently analyzed to determine the average and RMS wing deformation for the corresponding simulated flight condition.

\section{EXPERIMENT}

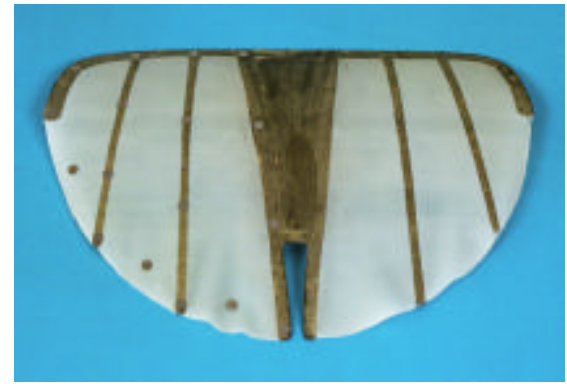

(a)

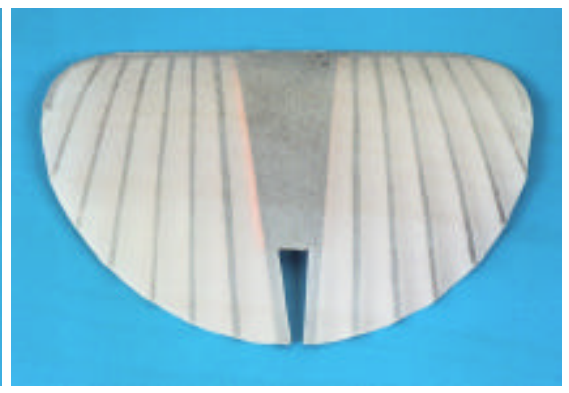

(b)

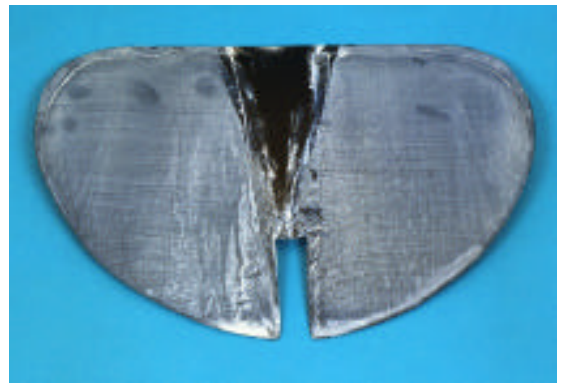

(c)

Figure 9: MAV wings studied in detail during March, 2001 tests: (a) 2-batten, latex covered wing, (b) 6-batten monofilm covered wing, (c) rigid composite wing (shown after oil flow studies) 
The three different wing designs shown in figures $9 \mathrm{a}-\mathrm{c}$ were studied in detail during the March, 2001 series of wind tunnel tests. All three wings had the same basic planform and camber, but varied in the number of battens, skin material, and stiffness. Each wing had a full span of $152 \mathrm{~mm}, 108 \mathrm{~mm}$ maximum chord length, and $1277 \mathrm{~mm}^{2}$ area. The wing shown in figure $9 \mathrm{a}$ is a 2-batten wing with a $0.1 \mathrm{~mm}$ latex skin. The wing shown in figure $9 \mathrm{~b}$ is a 6batten wing covered with a thin monofilm skin. The monofilm material, which was also used to cover the MAV fuselage, is a flexible Mylar-based film that is resistant to stretching. Use of the monofilm material in conjunction with the 6-batten construction provided a wing with significantly more stiffness compared to the 2-batten wing. The 6-batten wing was also more robust than the 2-batten wing, because the monofilm material was much more resistant to tearing and detachment from the battens compared to the $0.1 \mathrm{~mm}$ latex. The monofilm skin, which is naturally transparent, was very lightly dusted with white

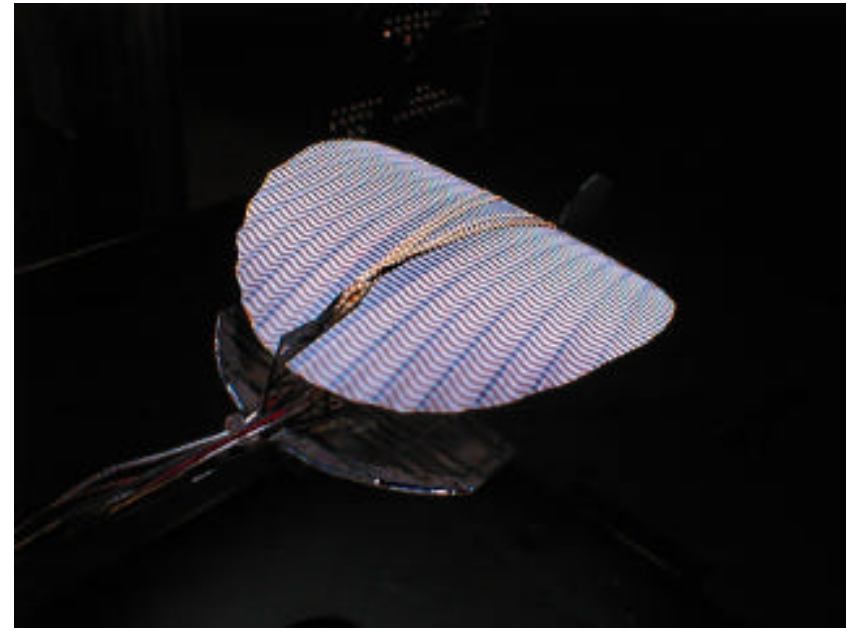

Figure 10: MAV with 6-batten monofilm covered wing and PMI projected grid lines. spray paint to provide a diffuse scattering surface for the PMI projected grid lines as shown in figure 10 . The wing shown in figure 9c is a 2-batten configuration covered with a graphite composite sheet. This wing was designed to be structurally rigid and not deform under aerodynamic loads. Thus aerodynamic performance data acquired for the MAV outfitted with the rigid wing could be used as a baseline for comparison to data obtained using wings with greater elasticity. The rigid wing, shown in figure 9c after oil flow visualization studies, was also dusted with white spray paint to aid in maintaining PMI projected grid line contrast. The wings were attached to the MAV fuselage using tightly stretched rubber bands. The wings had an approximate $+9^{\circ}$ angle of incidence when installed on the MAV, defined as the angle between the root chord line and the fuselage longitudinal axis.

During tunnel testing, PMI data were acquired for each wing while the vehicle AoA was swept from $-5^{\circ}$ to $+30^{\circ}$ in $2.5^{\circ}$ increments. The AoA sweeps were also repeated at different tunnel free stream velocities to examine velocity dependent trends in wing deformation and aerodynamic performance. PMI data of all three wings were acquired at tunnel velocities of $0 \mathrm{~m} / \mathrm{s}$ (wind off), $8.9 \mathrm{~m} / \mathrm{s}$ (dynamic pressure $\mathrm{Q}=47.9 \mathrm{~Pa}$ ) and $11.3 \mathrm{~m} / \mathrm{s}(\mathrm{Q}=76.6 \mathrm{~Pa}$ ). Additional $\mathrm{PMI}$ data were acquired for the 6-batten and rigid wings a tunnel velocity of $12.6 \mathrm{~m} / \mathrm{s}(\mathrm{Q}=95.7 \mathrm{~Pa})$. These velocities span the range of actual vehicle flight speeds.

\section{RESULTS}

Figures 11a-b show raw PMI images of the 2-batten and 6-batten wings respectively. The PMI measurement area for each wing has been outlined in white. The measurement area of the 2-batten wing covered a majority of the wing surface, except for some small regions near the trailing edge. The deformation of the latex skin in the clipped regions was so dynamic that the regions could not be measured. In fact, the latex skin near the trailing edge was observed to curl back over on itself in many of the PMI data images. Therefore these regions were simply masked out of the PMI processing. The measurement area for the 6-batten wing, as shown in figure $11 \mathrm{~b}$, included nearly the entire wing surface. Only some small fractions of the trailing edge required clipping. The measurement area for the rigid wing is not shown because it required no clipping whatsoever.

Figure 12 shows a subset of the wing deformation measurements obtained for the 2-batten and 6-batten wings at a tunnel speed of $11.3 \mathrm{~m} / \mathrm{s}$. The data were acquired in a powered flight condition with the MAV propeller spinning at approximately $18,000 \mathrm{rpm}$. The measurements represent the change in wing shape as a function of AoA. Positive deformation is defined as deformation occurring towards a viewer if the viewer were looking at the MAV wing top surface. The actual deformation data comprising figure 12 is smooth and continuous, but is shown in a 6-step color 


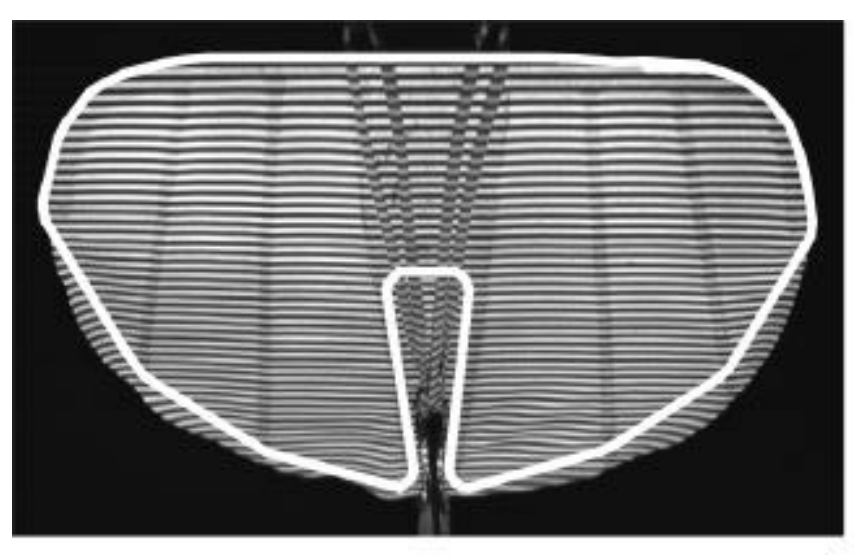

(a)

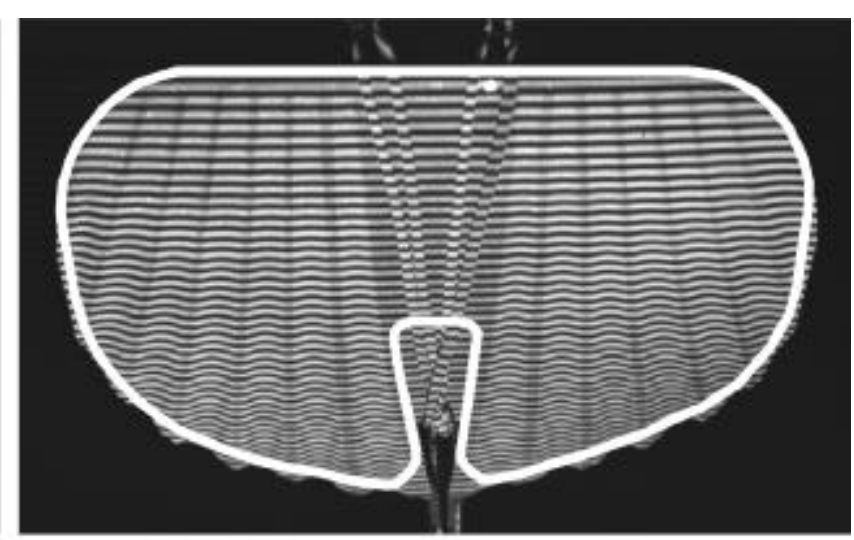

(b)

Figures 11a-b: Raw PMI data image of the (a) 2-batten wing, and (b) the 6-batten wing. The PMI measurement area for each wing is outlined in white.

palette for publication purposes. Breaks in the color palette correspond with deformation contours at a $1 \mathrm{~mm}$ contour interval. The contours exhibit significant scalloping for both the 2-batten and 6-batten wings, caused by deformation of the wing skin between the battens. The data indicate both wings continue to deform symmetrically, almost in a linear fashion, as AoA is increased. The 2-batten wing reached a maximum $7.6 \mathrm{~mm}$ deformation near the trailing edge at $+30^{\circ}$ AoA, while the 6-batten wing reached a maximum $5.1 \mathrm{~mm}$ deformation at the same condition. It is interesting to note that both wings have positive trailing edge deflections at $-5^{\circ}$ AoA. This is because the wings are mounted to the MAV fuselage with $\mathrm{a}+9^{\circ}$ angle of incidence, which aids in maintaining vehicle lift at negative AoA. The general trend in the data is that the wings continue to fill with air and deform as AoA increases, similar to a sail on a sailboat as the boat is tacked and steered perpendicular to the wind. This aeroelastic wing deformation results in a reduced effective AoA for the vehicle, resulting in a reduced vehicle stall angle ${ }^{5}$. Similar contour patterns at reduced magnitudes were observed over the same AoA range for the vehicle tested at a free stream velocity of $8.9 \mathrm{~m} / \mathrm{s}$.

Measurements of the wind-off absolute wing shape were also obtained in addition to wind-on deformation measurements. The wind-off wing shape was measured relative to the flat plate used for the PMI system calibration. These measurements fully describe the baseline wing geometry and camber, and are suitable for input to computational fluid dynamics (CFD) models. The complete wind-on wing geometry can thus be derived from the PMI measurements by adding the wind-on wing deformation measurements to the wind-off wing shape data. An example of this process is shown in figure 13. Figure 13a shows the wind-off wing shape for the 2-batten wing. The locations of the battens, wrinkles in the wing skin, and the rubber band used for mounting the wing to the fuselage can be seen. Figure 13b shows the average absolute wing shape for the 2-batten wing at a $11.3 \mathrm{~m} / \mathrm{s}$ tunnel free stream velocity and $+30^{\circ}$ AoA. Examination of the data shown in figure $13 \mathrm{~b}$ clearly shows how the MAV wing has deformed under aerodynamic load. The regions between the battens show significant scalloping of the wing skin, as shown in the deformation contour maps in figure 12. Displaying the data as a three-dimensional surface provides clear visualization of how the wing has deformed in resistance to the flow causing a reduction in wing camber and vehicle effective AoA. Data similar to that shown in figure 13 is currently being prepared for input into CFD models so that the geometry of each wing can be accurately modeled at all simulated flight conditions tested.

Figures 14-16 plot the total integrated deformation (TID) experienced by the 2-batten, 6-batten, and rigid wings for the different tunnel velocities and AoAs tested. The TID provides a single metric for examination of general trends in the deformation data and a means to achieve relative comparisons between wings. The wing TID at a given test condition was determined by integrating the PMI deformation measurements over the PMI measurement area. Linear extrapolation was used to adjust the TID data to compensate for slight differences in the PMI measurement area between wings, and to project the total deformation for the entire wing surface. Although this was a very minor correction, it permitted more accurate comparisons between wings. The data shown in figure 14 indicates the 2-batten wing deforms 


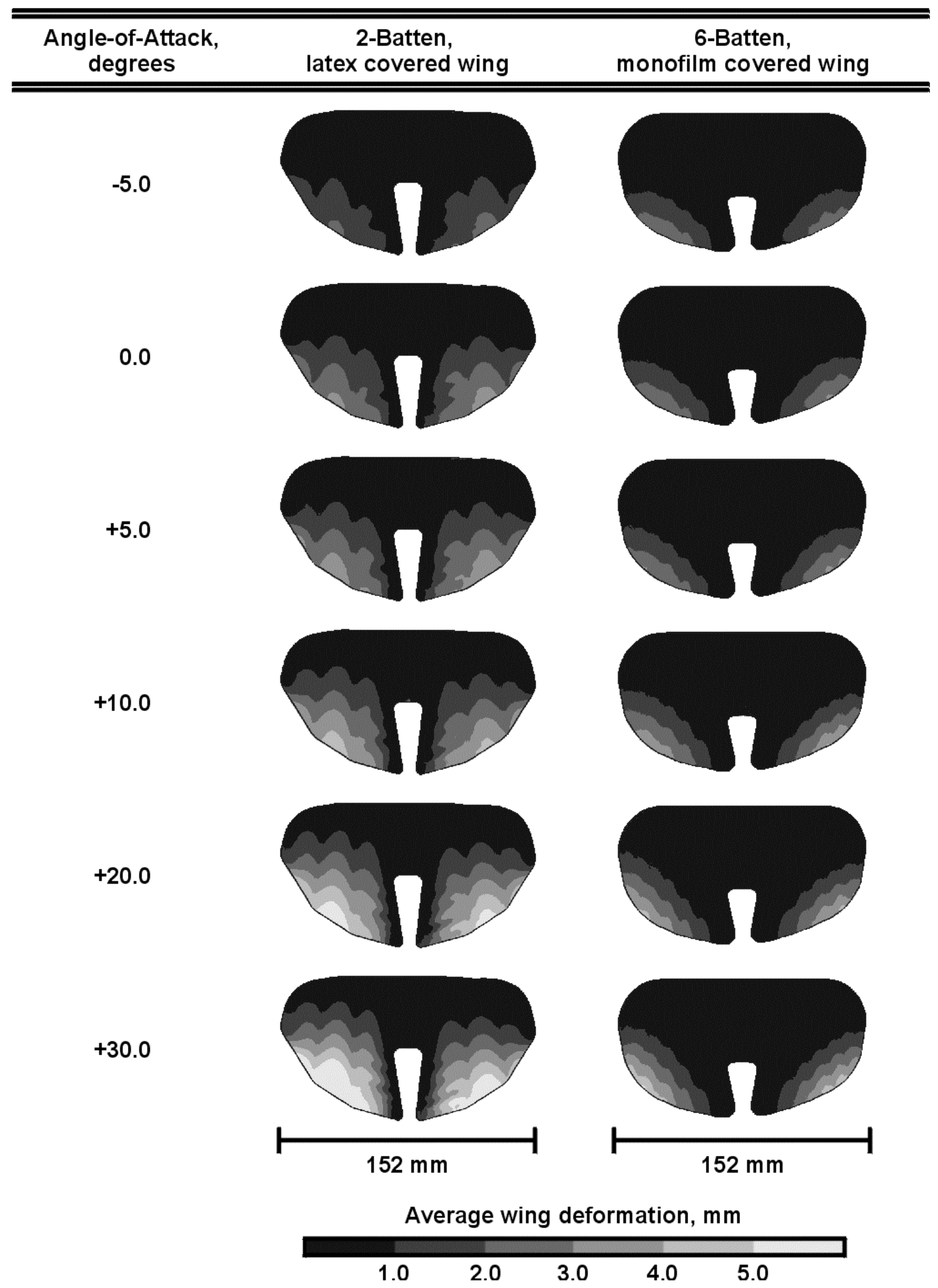

Figure 12: PMI-measured MAV wing deformation, tunnel free stream velocity $=11.3 \mathrm{~m} / \mathrm{s}$. 


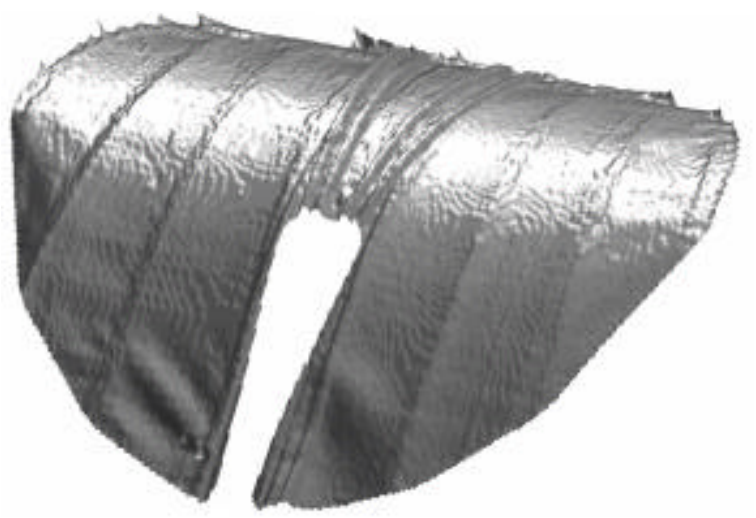

(a)

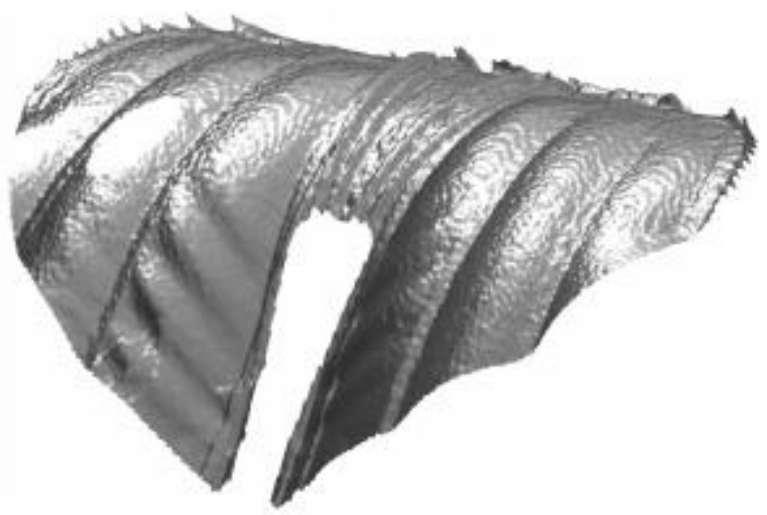

(b)

Figures 13a-b: Three-dimensional representation of 2-batten wing shape in (a) wind off, and (b) wind-on conditions.

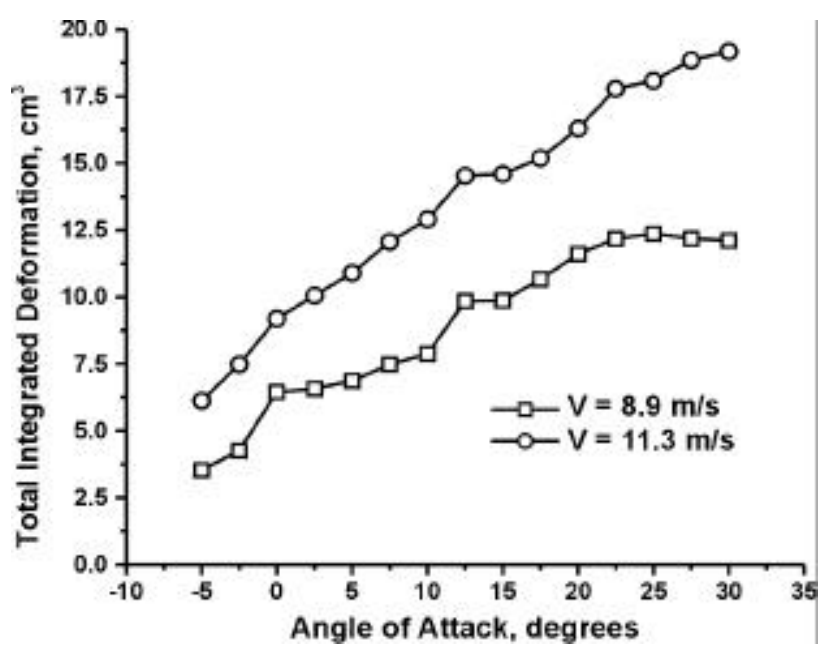

Figure 14: Total integrated deformation (TID) for the 2batten wing at tunnel speeds $\mathrm{V}=8.9$ and $11.3 \mathrm{~m} / \mathrm{s}$

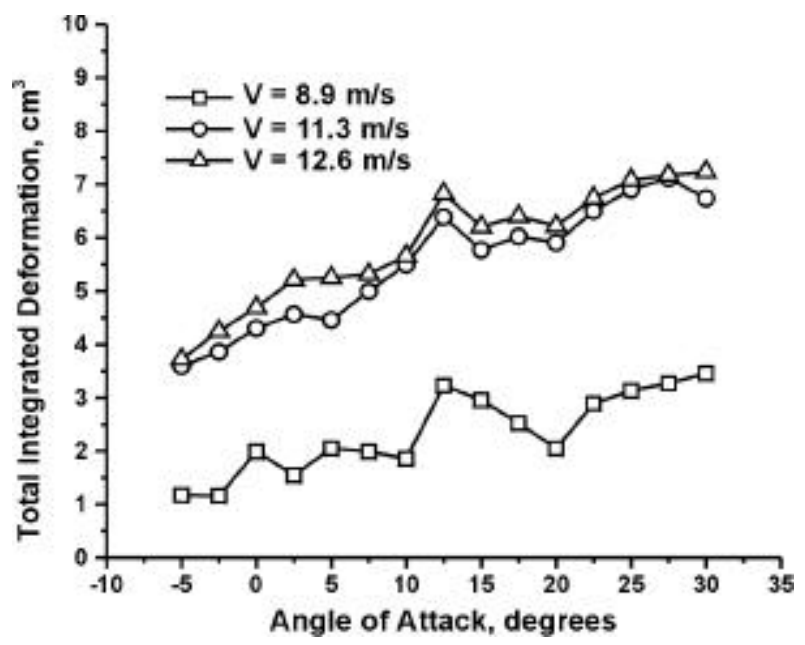

Figure 15: TID for the 6-batten wing at tunnel speeds $\mathrm{V}=8.9,11.3$, and $12.6 \mathrm{~m} / \mathrm{s}$

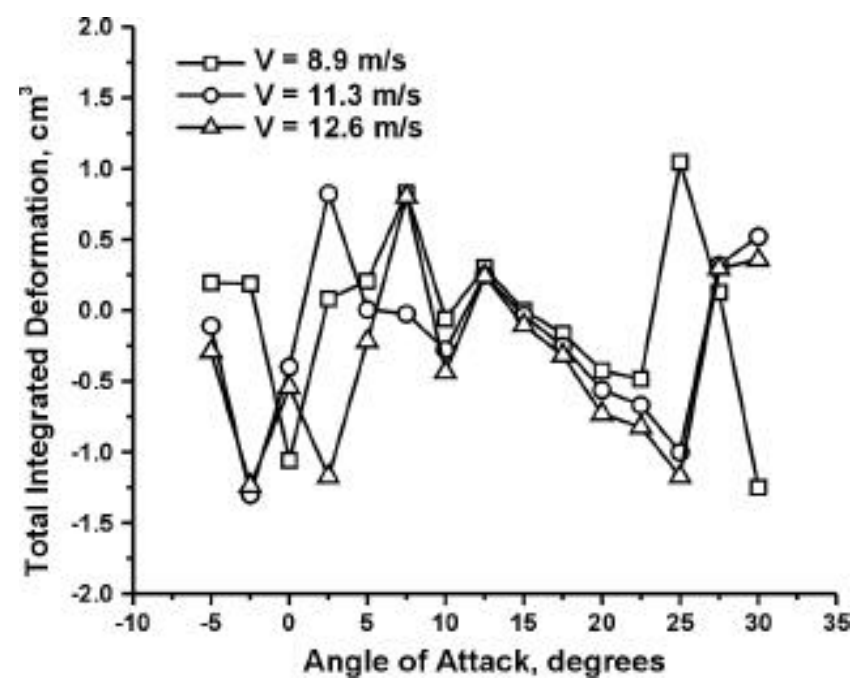

Figure 16: TID for the rigid wing at tunnel speeds $\mathrm{V}=8.9,11.3$, and $12.6 \mathrm{~m} / \mathrm{s}$ 
nearly linearly throughout the $-5^{\circ}$ to $+30^{\circ}$ AoA range for both tunnel speeds tested. The wing has an approximate $30 \%$ greater rate of deformation for the higher velocity case, and the maximum deformation at $+30^{\circ}$ AoA for the $11.3 \mathrm{~m} / \mathrm{s}$ case is nearly double the deformation occurring at the lower velocity. The TID data for the 6-batten wing is shown in figure 15. The 6-batten wing also deforms generally linearly as a function of AoA, but at a rate nearly $75 \%$ less than the 2-batten wing at $11.3 \mathrm{~m} / \mathrm{s}$ velocity. As with the 2-batten wing, the maximum deformation observed at $+30^{\circ}$ AoA doubles by changing the tunnel velocity from 8.9 to $11.3 \mathrm{~m} / \mathrm{s}$. However, the wing exhibits only slightly greater deformation at $12.6 \mathrm{~m} / \mathrm{s}$, suggesting the wing may be reaching its aeroelastic limit. The maximum deformation attained by the 6-batten wing is approximately 65\% less than that of the 2-batten wing. Figure 16 shows the TID for the rigid composite wing. The wing shows no distinguishable trend in deformation over the full AoA range, and the TID values are relatively small compared to the other wings. The negatively sloped trend from $+12.5^{\circ}$ to $+25^{\circ}$ AoA may be caused by small rigid body motions of the wing. Aerodynamically this wing has been shown to stall at $+12.5^{\circ 5}$. Since the wing can not deform to delay stall, any stresses on the wing are transferred to rubber bands mounting the wing to the fuselage, which will then stretch and allow rigid body motion. Surface contour plots similar to those shown in figure 12 for the rigid wing showed contours indicating slight rigid body motion about the vehicle roll axis, reaching a maximum $0.54^{\circ}$ roll at $+25^{\circ}$ AoA. However, these contour plots showed no evidence of any wing shape change at any of the conditions tested. Although this is not a very exciting result in terms of PMI measurements, it is significant in proving the wing maintained its shape throughout the test matrix and the baseline aerodynamic performance data were acquired with a truly rigid wing.

\section{PMI SYSTEM MEASUREMENT ACCURACY}

The PMI system measurement accuracy was assessed immediately following the initial system calibration. Accuracy was determined by rotating the calibration plate, in the same manner as was done for the calibration, to several arbitrary angles between $-10^{\circ}$ and $+10^{\circ}$ and acquiring PMI measurements of the plate. The measurements were then subtracted from the theoretical plate deformation at the same angles to establish an estimate for the PMI measurement error. Figure 17 shows the worst case error obtained over the surface of the plate, obtained at a plate rotation angle of $+8.33^{\circ}$. The measurement area used for the MAV wings is also identified. Figure 18 presents the histogram of the error data shown in figure 17. The histogram shows a positive bias and skew and errors up to $0.5 \mathrm{~mm}$. However, the large magnitude errors are near the bottom of the image and are outside the MAV measurement area. Errors within the measurement area lie primarily within the $\pm 0.2 \mathrm{~mm}$ bounds. Similar interrogations for the plate at other rotation angles showed errors typically within $\pm 0.1 \mathrm{~mm}$ within the MAV measurement area. Repositioning the PMI system as a function of vehicle AoA added another error source. Measurements in wind-off MAV wing shape over the $-5^{\circ}$ to $+30^{\circ}$ showed a typical maximum $\pm 0.2 \mathrm{~mm}$ variation in wing shape, appearing as an artificial roll angle. This systematic error was considered negligible and not corrected for in the final data. Thus the worst case accuracy of the PMI measurements is $\pm 0.4 \mathrm{~mm}$, with a nominal accuracy estimated at $\pm 0.25 \mathrm{~mm}$.

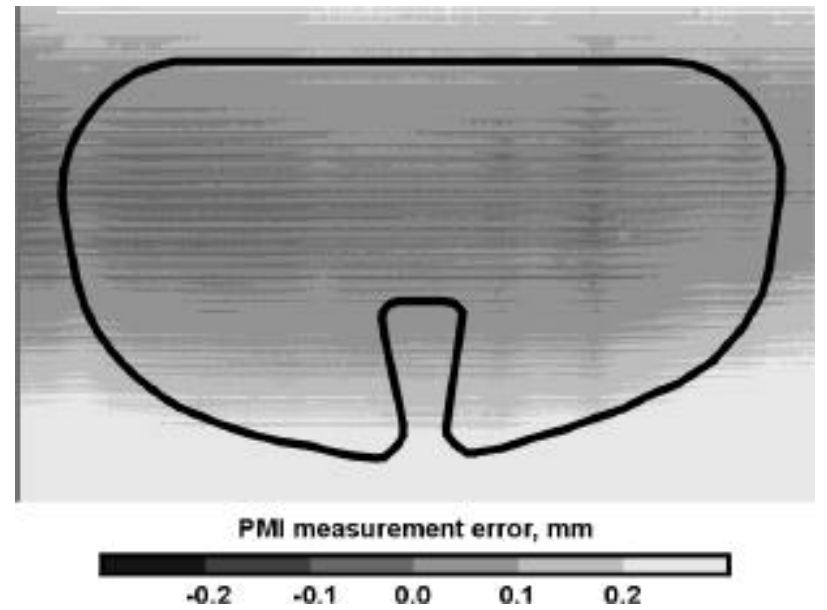

Figure 17: Worst case measurement error for rotation of the flat calibration plate

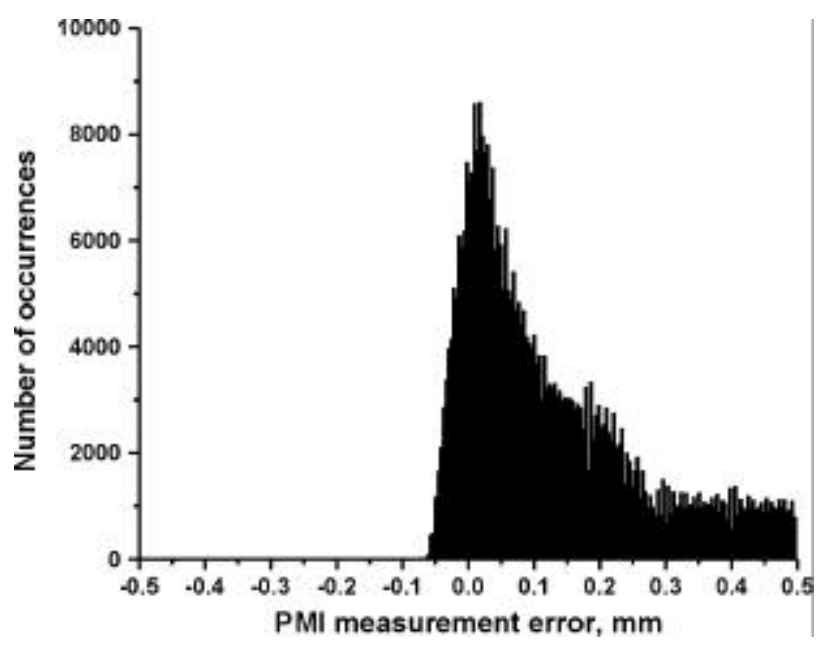

Figure 18: Histogram of the errors shown in figure 17 


\section{SUMMARY AND CONCLUSIONS}

A series of wind tunnel tests were conducted to investigate the benefits of flexible wings on micro air vehicle aerodynamic performance. Projection Moiré Interferometry (PMI) was used to quantify the aero-loaded wing shape for three different wing constructions of varying flexibility. The measurements fully describe the aero-loaded wing shape for a range of tunnel free stream velocity and vehicle angle-of-attack. The deformation measurement accuracy has been shown to be nominally $\pm 0.25 \mathrm{~mm}$. Generalized comparisons between wings were provided by examining the wing total integrated deformation as a function of simulated flight condition. Using this metric, it was found that a 6-batten, monofilm covered wing deformed approximately $65 \%$ less than a 2-batten, latex covered wing. A rigid, composite wing tested showed no change in shape throughout the test matrix, proving the aerodynamic performance data collected with this wing can be used as a baseline for comparison with other flexible wing designs. The complete database of wing shape measurements is currently being prepared for input into computational fluid dynamics (CFD) models, enabling accurate modeling of the wing shape at each simulated flight condition tested.

\section{ACKNOWLEDGEMENTS}

The authors wish to acknowledge Mr. Daniel J. Moyers of West Virginia University for investigating the implications of rotating / translating the PMI system, and Mr. Keith D. Grinstead of Swales Aerospace, Inc. for acquiring portions of the PMI data during the September, 2000 wind tunnel test.

\section{REFERENCES}

1. Mueller, T. J., editor, "Procedings of the Conference on Fixed, Flapping, and Rotary Wing Vehicles at Very Low Reynolds Numbers," Notre Dame University, Indiana, June 5-7, 2000.

2. Grasmeyer, J. M., and Keennon, M. T., "Development of the Black Widow Micro Air Vehicle", AIAA Paper No. AIAA-2001-0127, Presented at the $39^{\text {th }}$ AIAA Aerospace Sciences Meeting and Exhibit, Reno, NV, January, 2000.

3. Anon., University of Florida Micro Aerial Vehicle Research. http://aeroweb.aero.ufl.edu/microav/main.htm. June, 2001

4. Ifju, P. G., Ettinger, S., Jenkins, D. A., and Martinez, L., “Composite Materials for Micro Air Vehicles," Society for the Advancement of Materials and Process Engineering Annual Conference, Long Beach, CA, May 6-10, 2001.

5. Waszak, M. R., Jenkins, L. N., and Ifju, P., "Stability and Control Properties of an Aeroelastic Fixed Wing Micro Aerial Vehicle", AIAA Paper No. AIAA-2001-4005, Presented at the AIAA Atmospheric Flight Mechanics Conference, Montreal, Canada, August 6-9, 2001.

6. Fleming, G. A., and Gorton, S. A., "Measurement of rotorcraft blade deformation using Projection Moiré Interferometry", Journal of Shock and Vibration, Vol. 7, No. 3, 2000.

7. Wilbur, M. L., Mirick, P. H., Yeager, W. T., Langston, C. W., Cesnik, C. E., and Shin, S., "Vibratory loads reduction testing of the NASA / ARMY / MIT Active Twist Rotor", Presented at the American Helicopter Society $57^{\text {th }}$ Annual Forum, Washington, DC, May 9-11, 2001.

8. Fleming, G. A., and Burner, A. W., "Deformation measurements of smart aerodynamic surfaces", SPIE Paper No. 3783-25, Presented at the 44th Annual SPIE International Symposium on Optical Science, Engineering, and Instrumentation - Optical Diagnostics for Fluids/Heat/Combustion and Photomechanics for Solids, Denver, CO, July 18-23, 1999.

9. Fleming, G. A., Soto, H. L., South, B. W., and Bartram, S. M., "Advances in Projection Moiré Interferometry Development for Large Wind Tunnel Applications", SAE Paper No. 1999-01-5598, Presented at the SAE World Aviation Congress, San Francisco, CA, October 19-21, 1999.

10. Pirodda, L., "Shadow and projection moiré techniques for absolute or relative mapping of surface shapes", Optical Engineering 21(4), pp. 640 - 649, July/August 1982.

11. Sellers, W. L., III and Kjelgaard, S. O.: The Basic Aerodynamics Research Tunnel - A Facility Dedicated to Code Validation, AIAA-88-1997, May, 1988.

12. Washburn, A. E.: The Effect of Freestream Turbulence on the Vortical Flow over a Delta Wing, Master of Science Thesis, George Washington University, December, 1990.

13. Jenkins, L. N.: An Experimental Investigation of the Flow Over a Notchback Automobile Configuration, Master of Science Thesis, George Washington University, January, 1999. 